Gaining competence in rarely performed interventional procedures is difficult. We present here a pig model for endoscopic drainage of pancreatic pseudocysts and describe its preparation and the procedure. Endoscopic drainage of pancreatic pseudocysts through the wall of the gastrointestinal tract is a challenging procedure for any endoscopist because of the need to cross the peritoneal cavity [1]. The caseload of many endoscopy centres is usually relatively small and may only amount to five to 20 procedures per year, and the opportunities for teaching and learning are limited. We considered that the Erlangen Active Simulator for Interventional Endoscopy (EASIE) model for endoscopic retrograde cholangiopancreatography designed by Hochberger and Maiss might be suitable [2]. This model consists of a pig esophagus, stomach and duodenum with the ligamentum hepatoduodenale, the whole biliary system and the liver, lying in a plastic box; it does not have a pancreas because the pig pancreas does not drain via the main papilla.

We placed the gallbladder next to the anterior duodenal wall and fixed it with four sutures, as shown in Figure 1, mimicking a pancreatic head cyst with duodenal bulging. We had filled the biliary system via the papillary route earlier with water mixed with blue dye for better bulging and better visualization of the draining fluid. The correct site for puncturing can either be detected through the bulging region or during endoscopic ultrasound; alternatively, it may be located at the centre of the four sutures when the stitches have penetrated the whole thickness of the gastrointestinal wall. A neutral electrode was placed beneath the model. We used a TJF-160R endoscope (Olympus Co., Tokyo, Japan) and the 10-Fr Cremer pseudocyst drainage set in two instances. The set consists of a needle-knife and a coaxial conic-tip cutting device. The intervention ends with the placement of a double-pigtail stent using the Seldinger technique (Figure 2,3 ). On both occasions the simulation worked well. The only

\title{
Adaptation of the Erlangen Active Simulator for Interventional Endoscopy (EASIE) Model for Transmural Pancreatic Pseudocyst Drainage
}
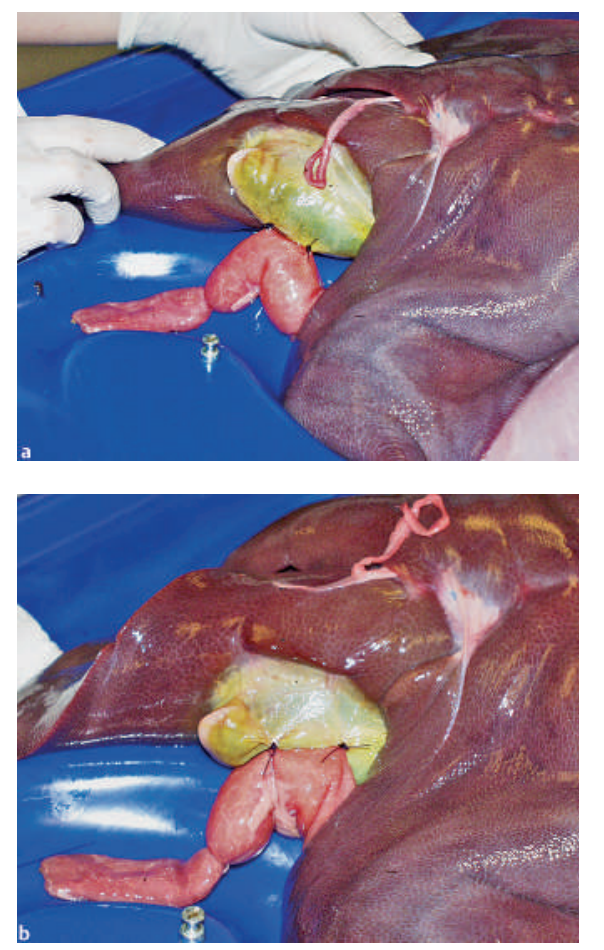

Figure 1 In order to simulate a pancreatic head cyst with duodenal bulging, the gallbladder was placed next to to the anterior duodenal wall and fixed using four stitches. These photographs show the first two stitches (a) and the third and fourth stitches (b).

problem was that after enlargement of the duodenobiliary fistula the duodenum lost almost all the inflated air. A device with a smaller diameter (e.g. 8 Fr) may work better.

Attachment of a prefilled gallbladder to the duodenum in the EASIE model with sutures appears to create a valuable hands-on model for endoscopic pancreatic pseudocyst drainage.

\section{R. Schöfl, B. Buchmeier, G. Hauder}

Department of Gastroenterology,

Elisabethinen Hospital, Linz, Austria

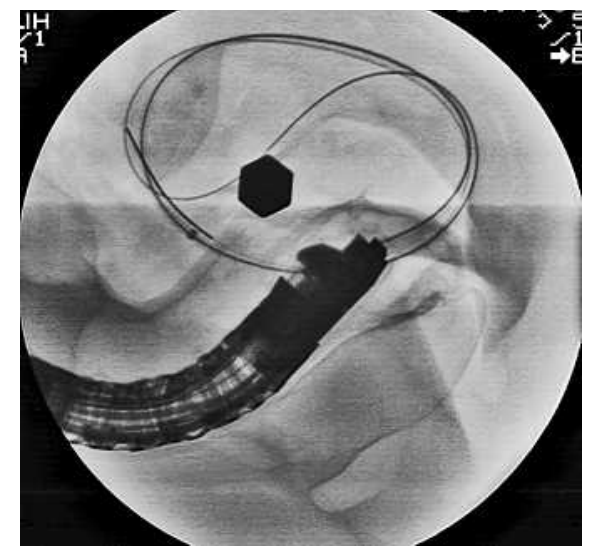

Figure 2 A double-pigtail stent was placed using the Seldinger technique. This fluoroscopic view shows the guide wire placed into the gallbladder.

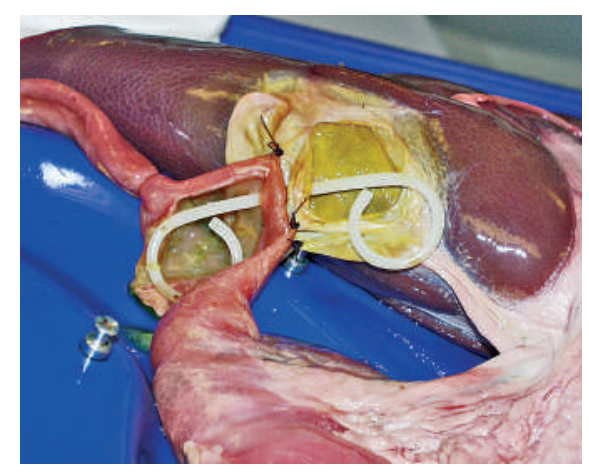

Figure 3 The duodenum and gallbladder are opened after the procedure, demonstrating the correct positioning.

\section{References}

${ }^{1}$ Cremer M, Deviere J, Engholm L. Endoscopic management of cysts and pseudocysts in chronic pancreatitis: longterm follow-up after 7 years of experience. Gastrointest Endosc 1989; 35: 1 - 9

${ }^{2}$ Hochberger J, Maiss J, Hahn EG. The Use of Simulators for Training in GI Endoscopy. Endoscopy 2002; 34: 727-729

\section{Corresponding Author}

\section{R. Schöfl, M.D.}

Department of Internal Medicine IV Gastroenterology and Hepatology, Elisabethinen Hospital, Fadinger Straße 1, 